\title{
An efficacy comparison of anti-vascular growth factor agents and laser photocoagulation in diabetic macular edema: a network meta-analysis incorporating individual patient-level data
}

Dominic Muston ${ }^{1 *}$, Jean-Francois Korobelnik ${ }^{2,3}$, Tim Reason ${ }^{4}$, Neil Hawkins ${ }^{5}$, Ismini Chatzitheofilou ${ }^{4}$, Fay Ryan ${ }^{4}$ and Peter K. Kaiser ${ }^{6}$

\begin{abstract}
Background: This was an updated network meta-analysis (NMA) of anti-vascular endothelial growth factor (VEGF) agents and laser photocoagulation in patients with diabetic macular edema (DME). Unlike previous NMA that used meta-regression to account for potential confounding by systematic variation in treatment effect modifiers across studies, this update incorporated individual patient-level data (IPD) regression to provide more robust adjustment.

Methods: An updated review was conducted to identify randomised controlled trials for inclusion in a Bayesian NMA. The network included intravitreal aflibercept (IVT-AFL) 2 mg bimonthly (2q8) after 5 initial doses, ranibizumab $0.5 \mathrm{mg}$ as-needed (PRN), ranibizumab $0.5 \mathrm{mg}$ treat-and-extend (T\&E), and laser photocoagulation. Outcomes included in the analysis were change in best-corrected visual acuity (BCVA), measured using an Early Treatment Diabetic Retinopathy Study (ETDRS) chart, and patients with $\geq 10$ and $\geq 15$ ETDRS letter gains/losses at 12 months. Analyses were performed using networks restricted to IPD-only and IPD and aggregate data with (i) no covariable adjustment, (ii) covariable adjustment for baseline BVCA assuming common interaction effects (against reference treatment), and (iii) covariable adjustments specific to each treatment comparison (restricted to IPD-only network).

Results: Thirteen trials were included in the analysis. IVT-AFL $2 q 8$ was superior to laser in all analyses. IVT-AFL 2q8 showed strong evidence of superiority ( $95 \%$ credible interval [Crl] did not cross null) versus ranibizumab $0.5 \mathrm{mg}$ PRN for mean change in BCVA (mean difference 5.20,95\% Crl 1.90-8.52 ETDRS letters), $\geq 15$ ETDRS letter gain (odds ratio [OR] 2.30, 95\% Crl 1.12-4.20), and $\geq 10$ ETDRS letter loss (OR 0.25, 95\% Crl 0.05-0.74) (IPD and aggregate random-effects model with baseline BCVA adjustment). IVT-AFL 2 q8 was not superior to ranibizumab $0.5 \mathrm{mg}$ T\&E for mean change in BCVA (mean difference 5.15, 95\% Crl -0.26-10.61 ETDRS letters) (IPD and aggregate random-effects model).
\end{abstract}

Conclusions: This NMA, which incorporated IPD to improve analytic robustness, showed evidence of superiority of IVT-AFL 2q8 to laser and ranibizumab $0.5 \mathrm{mg}$ PRN. These results were irrespective of adjustment for baseline BCVA.

Keywords: Intravitreal aflibercept, Diabetic macular edema, Intravitreal ranibizumab, Meta-analysis

\footnotetext{
*Correspondence: dom.muston@gmail.com

'Bayer US LLC, Whippany, NJ, USA

Full list of author information is available at the end of the article
}

(c) The Author(s). 2018 Open Access This article is distributed under the terms of the Creative Commons Attribution 4.0 International License (http://creativecommons.org/licenses/by/4.0/), which permits unrestricted use, distribution, and reproduction in any medium, provided you give appropriate credit to the original author(s) and the source, provide a link to the Creative Commons license, and indicate if changes were made. The Creative Commons Public Domain Dedication waiver (http://creativecommons.org/publicdomain/zero/1.0/) applies to the data made available in this article, unless otherwise stated. 


\section{Background}

Diabetic macular edema (DME) is the leading cause of vision loss in patients with diabetic retinopathy [1]. Treatments include anti-vascular endothelial growth factor (VEGF) agents, laser, steroids, and surgery. Anti-VEGF agents are currently standard of care in DME treatment. These agents are known to target underlying abnormalities in the VEGF signalling cascade, which is a primary pathway in DME progression [2]. Anti-VEGF agents reduce the incidence of legal blindness, and their long-term efficacy and safety have been proven in numerous randomised trials [3-8].

Direct randomised comparisons of anti-VEGF agents in DME, however, are limited to the Diabetic Retinopathy Clinical Research Network (DRCR.net) Protocol T trial [4, 8], which only included ranibizumab at $0.3 \mathrm{mg}$ (US dose) and did not include ranibizumab at $0.5 \mathrm{mg}$ (EU dose) and is therefore not directly relevant to retina practice outside the United States. Many comparator DME trials have also used mean change in best-corrected visual acuity (BCVA; Early Treatment Diabetic Retinopathy Study [ETDRS] letters) after 12 months as a primary efficacy measure, but differences in baseline BCVA between trials, a likely treatment effect modifier $[4,8]$, may confound indirect comparisons.

Previous network meta-analyses (NMA) of anti-VEGF agents [9-11] have attempted to adjust for baseline BCVA using aggregate data. These analyses provide useful data for decision-making, but meta-regression based on aggregate data may not mirror effects at the patient level and could also be confounded by non-linear covariable effects and other sources of heterogeneity between trials that are not accounted for by the variables included in the meta-regression $[12,13]$. This bias is known as ecological bias.

The European Medicines Agency has recently issued a policy with a primary objective of making clinical reports and individual patient-level data (IPD) available to enable public scrutiny and allow for the application of new knowledge in future research [14]. Within-trial regression analysis based on IPD provides more robust adjustment for differences in BCVA between trials. However, to our knowledge, no published NMA has incorporated IPD.

The objective of this NMA was to perform an updated indirect comparison of the efficacy of approved anti-VEGF regimens and laser photocoagulation in DME based on mean change in BCVA and gain or loss of letters at 12 months. The NMA incorporated both aggregate data and IPD, where available. Different regimens, including fixed, as-needed (PRN), and treat-and-extend (T\&E), were also included. These regimens have not been incorporated in detail in previous NMAs.

\section{Methods}

\section{Search strategy}

The literature search was updated from a previous systematic literature review performed in February 2015
[9]. This updated literature review identified all relevant randomised controlled trials (RCTs) that were published from February 2015 to December 2016 using the same search strategy that was described in the previous publication [9]. An additional search of ClinicalTrials.gov (from January 2015 to December 2016) was also performed to identify any new trials. This was performed by searching for 'diabetic macular edema phase III RCT' to be consistent with the previous analysis. As summarized in Fig. 1, the analysis presented here uses data from all studies identified above.

\section{Inclusion criteria}

Trials were screened using the populations, interventions, comparators, outcomes, and study design (PICOS) criteria as defined in the previous publication [9]. This approach also applied to the results from the ClinicalTrials.gov search. Only trials that reported the randomised controlled results at 12 months for mean change from baseline in BCVA (ETDRS letters) (continuous outcome), and the proportion of patients achieving gain/ loss of $\geq 10$ and $\geq 15$ ETDRS letters (binary outcomes) were included.

Treatment regimens of interest for European practice were IVT-AFL $2 \mathrm{mg}$ every 8 weeks (2q8) after 5 initial doses, ranibizumab $0.5 \mathrm{mg} \mathrm{PRN}$, ranibizumab $0.5 \mathrm{mg}$ $\mathrm{T} \& \mathrm{E}$, and laser photocoagulation. Because it is not licensed for ophthalmic use, bevacizumab was not included in the analysis. Treatments were grouped according to dose, number of loading doses, regimen, which could be proactive (fixed), reactive (PRN), or proactive/reactive (T\&E), and median number of injections. Treatment regimens for ranibizumab and IVT-AFL were not the same because treatment regimens were determined by the individual study sponsors. These groupings were used for treatment classification in the NMA. All included trials were compliant with the Declaration of Helsinki, had protocols approved by relevant countryand trial-specific institutional review boards/independent ethics committees, and enrolled patients who provided written informed consent for participation.

\section{Data extraction}

Data extraction was based on methods described previously [9]. Missing standard errors (SEs) for the change in continuous outcome were imputed using the following equation [15]:

$$
S E_{\text {change }}=\frac{\sqrt{S D_{\text {baseline }}^{2}+S D_{\text {final }}^{2}-\left(2 \times \text { correlation } \times S D_{\text {baseline }} \times S D_{\text {final }}\right)}}{\sqrt{\text { sample size }}}
$$

Where $S D_{\text {final }}$ was not available, $S D_{\text {baseline }}$ was used in place of $S D_{\text {final }}$. For the correlation, 0.5 was used in the 


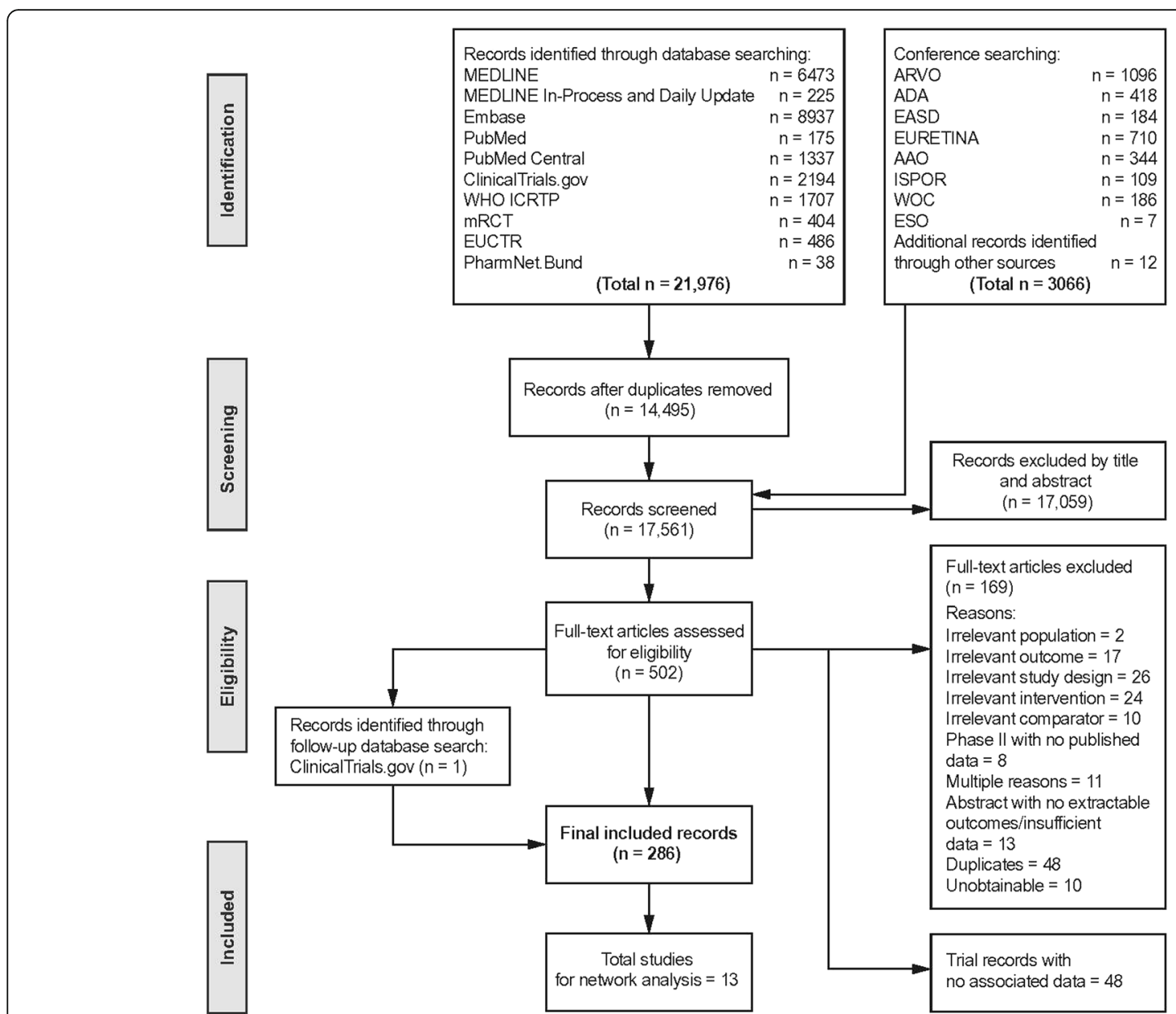

Fig. 1 Flow chart summarizing the literature search

formula above; this has been described as a conservative assumption [16]. Standard deviation (SD) and 95\% confidence intervals (CIs) were converted to SEs based on established formulas ([15]. If CI, SD, and SE were not reported and only $P$ values were available, it was assumed that the $P$ values were calculated using a Wald test. The SE was then calculated by rearranging the formula for calculating $P$ values for normally distributed variables.

\section{Analyses}

The trials were used to form a Bayesian NMA for continuous and binary outcomes. Analyses were performed using IPD-only and IPD and aggregate data networks with (i) no covariable adjustment, (ii) covariable adjustment for baseline BVCA assuming common interaction effects (against reference treatment), and (iii) covariable adjustments specific to each treatment comparison (IPD-only). Both fixed-effect and random-effects models were fitted.

Baseline BCVA was identified as the main treatment effect modifier (covariable) for vision-related outcomes, and was controlled for in the NMA. Based on previous analysis of IPD from the VIVID-DME and VISTA-DME trials, other potential covariables (diabetic retinopathy severity scale, prior anti-VEGF treatment, baseline glycated haemoglobin, blood pressure treatment, and cataracts) did not have a significant impact on IVT-AFL treatment effects and were not included [17-21]. Baseline central retinal thickness (CRT) was shown to be a potential treatment effect modifier on vision-related outcomes when receiving IVT-AFL treatment [22]; however, it was excluded as it was found to be highly correlated with baseline BCVA, which would have resulted in co-linearity in the model. An additional file contains further information on the models and covariable adjustment (see Additional file 1: Appendix 1).

The analyses were conducted with OpenBUGS version 3.2.3 using Monte Carlo chain simulations to calculate posterior distributions for the parameters of interest. A Monte Carlo error of less than 5\% of the posterior SD indicated acceptable simulation error [23]. The absolute and relative model fits were assessed using residual deviance 
and deviance information criterion statistics. Convergence was assessed by visual inspection of caterpillar and probability density plots and by running analyses using 3 separate chains to ensure that all chains converged to the same distribution. We ran all models with a burn-in of 50,000 and 200,000 iterations.

All baseline and intervention parameters were given vague normal (mean $=0, \mathrm{SD}=1000$ ) distributions, an appropriately large range given the scale of measurement. A binomial likelihood with logit link function was used for binary data, and a normal likelihood with identity link function was used for continuous data. The OpenBUGS codes used were adapted from previously published codes [24]. This allowed the code to be easily adapted to include IPD. The methodology also followed guidance from the International Society for Pharmacoeconomics and Outcomes Research Task Force on Indirect Treatment Comparisons [25, 26].

Binary outcomes were reported as odds ratios (ORs), and the continuous outcome was reported as the mean treatment difference (ETDRS letters). Uncertainty was reported using 95\% credible intervals (CrIs). IVT-AFL 2q8 was chosen as the reference treatment so that it could be compared with all treatments of interest (ie, ranibizumab $0.5 \mathrm{mg} \mathrm{PRN}$, ranibizumab $0.5 \mathrm{mg} \mathrm{T} \& \mathrm{E}$, and laser photocoagulation).

\section{Results}

\section{Networks}

A total of 13 trials were identified as eligible for inclusion $[4-7,27-33]$. The treatment regimens in these trials were classified and included in the NMA as summarised in Additional file 2: Appendix 2; an overview of the outcomes reported in these trials is summarised in Additional file 3: Appendix 3. Thirteen trials provided continuous outcome data (ie, mean change in BCVA) and 10 trials provided binary outcome data (ie, proportion of patients with loss/gain of $\geq 10$ and/or $\geq 15$ ETDRS letters) as summarised in Table 1. Baseline BCVA was adjusted for by incorporating IPD from 5 trials and aggregate data from 8 trials. IPD-only and IPD and aggregate data networks were developed using these data, which are summarised in Fig. 2.

In all analyses, IVT-AFL 2q8 was shown to be superior to laser. Overall, the means for the coefficients describing the interactions effects were negative (see Additional file 1: Appendix 1) indicating that patients with lower BCVA at baseline had a greater response to treatment. Only random-effects IPD and aggregate models are described here.

\section{Mean change in BCVA (continuous outcome)}

The results for the mean change in BCVA (ETDRS letters) from the analysis of the IPD-only and IPD and aggregate data networks are shown in Table 2. IVT-AFL
Table 1 Overview of trial data included in each analysis [4-8, 27-32]

\begin{tabular}{ll}
\hline IPD and aggregate network & IPD-only network \\
\hline DRCR.net Protocol I & DRCR.net Protocol I \\
DRCR.net Protocol J & DRCR.net Protocol J \\
DRCR.net Protocol T & VISTA-DME \\
LUCIDATE $^{\text {a }}$ & VIVID-DME \\
RESPOND $^{\text {b }}$ & VIVID-EAST \\
RESTORE & \\
RETAIN & \\
REVEAL & \\
RIDE & \\
RISE & \\
VISTA-DME & \\
VIVID-DME & \\
VIVID-EAST &
\end{tabular}

aLUCIDATE, RISE and RIDE did not provide data for letter gains/losses

${ }^{\text {b} R E S P O N D ~ d i d ~ n o t ~ p r o v i d e ~ d a t a ~ f o r ~ l e t t e r ~ l o s s e s ~}$

2q8 showed strong evidence of superiority (95\% CrI did not cross 0 ) versus laser, ranibizumab $0.5 \mathrm{mg} \mathrm{PRN}$, and ranibizumab $0.5 \mathrm{mg}$ PRN with laser in the IPD and aggregate networks (all covariable adjustments) for mean change in BCVA. The mean difference $(95 \% \mathrm{CrI})$ in BCVA (ETDRS letters) for IVT-AFL 2q8 versus ranibizumab $0.5 \mathrm{mg}$ PRN was 5.15 (1.82-8.54) (no covariable adjustment) and 5.20 (1.90-8.52) (common covariable adjustment). IVT-AFL $2 \mathrm{q} 8$ was not superior to ranibizumab $0.5 \mathrm{mg}$ T\&E for mean change in BCVA (mean difference 5.15; 95\% CrI -0.26-10.61 ETDRS letters) (IPD and aggregate random-effects model).

\section{Proportion of patients gaining or losing $\geq 10$ and $\geq 15$ ETDRS letters (binary outcomes)}

The IPD-only and IPD and aggregate data network results for letter gains are shown in Table 3. IVT-AFL 2q8 showed strong evidence of superiority (95\% CrI did not cross 1 ) for the proportion of patients gaining $\geq 15$ ETDRS letters versus laser, ranibizumab $0.5 \mathrm{mg}$ PRN, ranibizumab $0.5 \mathrm{mg}$ PRN with laser, and ranibizumab $0.5 \mathrm{mg}$ T\&E with laser in the IPD and aggregate networks (all covariable adjustments). The OR ( $95 \% \mathrm{CrI}$ ) for gaining $\geq 15$ ETDRS letters for IVT-AFL 2q8 versus ranibizumab $0.5 \mathrm{mg}$ PRN was 2.08 (1.06-3.70) (no covariable adjustment) and 2.30 (1.12-4.20) (common covariable adjustment).

The IPD-only and IPD and aggregate data network results for the proportion of patients losing $\geq 10$ and $\geq 15$ ETDRS letters are shown in Table 4. IVT-AFL 2q8 showed strong evidence of superiority ( $95 \% \mathrm{Crl}$ did not cross 1) for reducing the proportion of patients losing $\geq 10$ or $\geq 15$ ETDRS letters versus laser. IVT-AFL 2 q8 was also superior to ranibizumab $0.5 \mathrm{mg}$ PRN, ranibizumab $0.5 \mathrm{mg}$ PRN with laser, and ranibizumab $0.5 \mathrm{mg}$ 
a
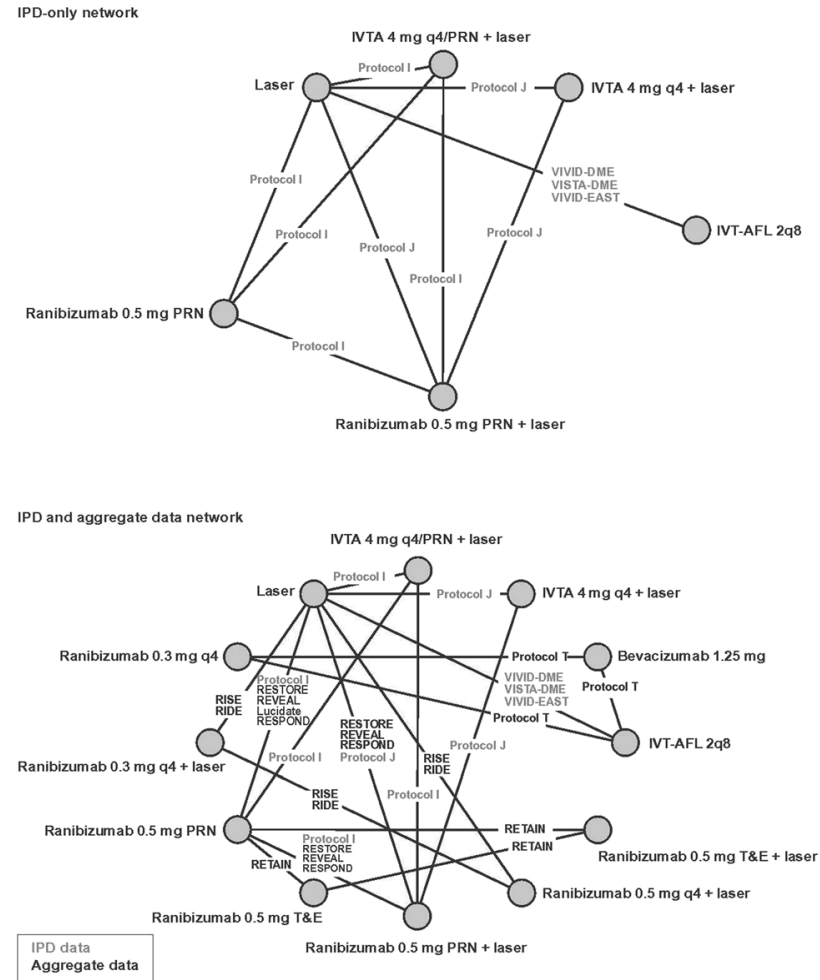

b

IPD-only network

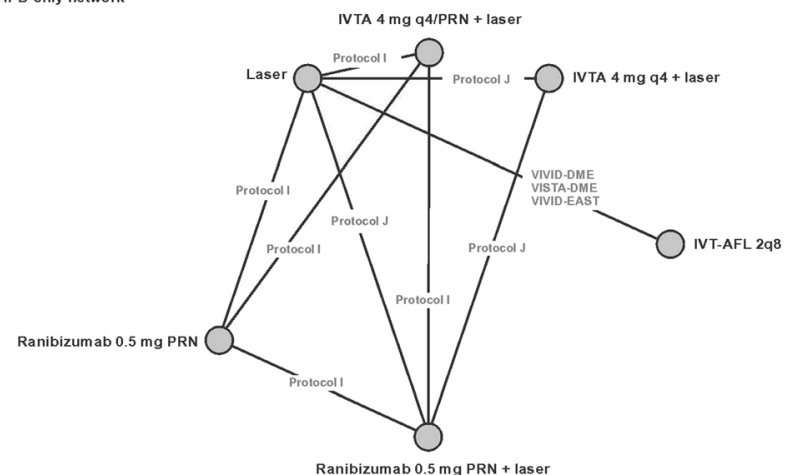

IPD and aggregate data network $\quad$ INTA 4 mg q4/PRN + laser

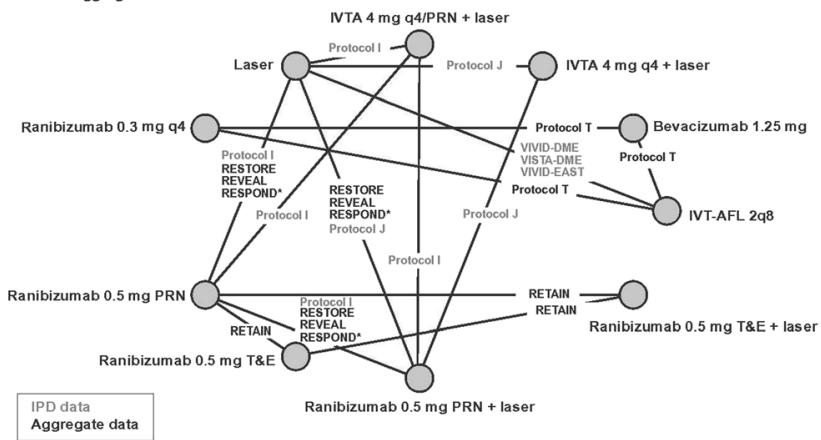

Fig. 2 (See legend on next page.) 
(See figure on previous page.)

Fig. 2 IPD-only and IPD and aggregate data networks for (a) mean change in BCVA (continuous outcome) and (b) gain/loss of $\geq 10$ and $\geq 15$ ETDRS letters (binary outcomes). 2q8, every 8 weeks; BCVA, best-corrected visual acuity; ETDRS, Early Treatment Diabetic Retinopathy Study; IPD, individual patient-level data; IVTA, intravitreal triamcinolone acetonide; IVT-AFL, intravitreal aflibercept; PRN, as-needed; q4, every 4 weeks; T\&E, treat-and-extend. ${ }^{2}$ RESPOND did not provide data for letter losses. Note: outcomes for ranibizumab $0.3 \mathrm{mg}$ (US dose), intravitreal triamcinolone acetonide and bevacizumab are not reported

T\&E in the IPD and aggregate networks (all covariable adjustments) for reducing $\geq 10$ ETDRS letter losses. The OR (95\% Crl) for losing $\geq 10$ ETDRS letters for IVT-AFL 2q8 versus ranibizumab $0.5 \mathrm{mg} \mathrm{T} \& \mathrm{E}$ was $0.19(0.00-$ $0.96)$ (no covariable adjustment) and $0.21(0.00-0.97)$ (common covariable adjustment). However, a number of OR values calculated were implausible, which may be attributable to reduced evidence supporting comparison for ETDRS letters in Table 3 and Table 4. These implausible values are limited to certain analyses of the IPD-only dataset, and may be a result of the methodology employed.

Table 2 IPD-only and IPD and aggregate network results for mean change in BCVA (ETDRS letters)

\begin{tabular}{|c|c|c|c|c|c|c|}
\hline \multirow[b]{2}{*}{ Comparison } & \multirow[b]{2}{*}{ Covariable adjustment } & \multirow[b]{2}{*}{ Model } & \multicolumn{2}{|l|}{ IPD-only network } & \multicolumn{2}{|c|}{ IPD and aggregate network } \\
\hline & & & Mean difference & $95 \% \mathrm{Crl}$ & Mean difference & $95 \% \mathrm{Crl}$ \\
\hline \multirow[t]{6}{*}{ IVT-AFL 2q8 vs laser } & None & Fixed & $+10.32^{\mathrm{a}}$ & $8.31-12.34$ & $+10.32^{a}$ & $8.30-12.35$ \\
\hline & & Random & $+10.47^{\mathrm{a}}$ & $6.92-14.16$ & $+10.45^{a}$ & $7.81-13.12$ \\
\hline & Common (baseline BCVA) & Fixed & $+10.30^{\mathrm{a}}$ & $8.82-11.78$ & $+10.27^{\mathrm{a}}$ & $8.79-11.74$ \\
\hline & & Random & $+10.54^{a}$ & $7.07-14.42$ & $+10.47^{a}$ & 7.88-13.11 \\
\hline & Treatment-specific & Fixed & $+10.48^{a}$ & $8.99-11.97$ & - & - \\
\hline & & Random & $+10.65^{a}$ & $7.13-14.34$ & & \\
\hline \multirow[t]{6}{*}{ IVT-AFL 2q8 vs ranibizumab 0.5 mg PRN } & None & Fixed & $+3.92^{\mathrm{a}}$ & $0.60-7.24$ & $+4.42^{\mathrm{a}}$ & $2.07-6.76$ \\
\hline & & Random & +3.47 & $-3.14-10.44$ & $+5.15^{\mathrm{a}}$ & $1.82-8.54$ \\
\hline & Common (baseline BCVA) & Fixed & $+3.14^{\mathrm{a}}$ & $0.48-5.80$ & $+4.95^{\mathrm{a}}$ & $3.09-6.83$ \\
\hline & & Random & +3.54 & $-2.93-10.81$ & $+5.20^{\mathrm{a}}$ & $1.90-8.52$ \\
\hline & Treatment-specific & Fixed & $+3.09^{\mathrm{a}}$ & $0.44-5.72$ & - & - \\
\hline & & Random & +3.38 & $-3.20-10.18$ & & \\
\hline \multirow[t]{6}{*}{ IVT-AFL 2 q8 vs ranibizumab 0.5 mg PRN + laser } & None & Fixed & $+5.96^{\mathrm{a}}$ & $2.65-9.25$ & $+5.17^{\mathrm{a}}$ & $2.73-7.61$ \\
\hline & & Random & $+6.05^{\mathrm{a}}$ & $0.45-12.19$ & $+5.95^{\mathrm{a}}$ & $2.60-9.37$ \\
\hline & Common (baseline BCVA) & Fixed & $+5.71^{\mathrm{a}}$ & $3.11-8.29$ & $+5.77^{\mathrm{a}}$ & $3.81-7.74$ \\
\hline & & Random & $+6.16^{\mathrm{a}}$ & $0.67-12.55$ & $+6.00^{\mathrm{a}}$ & $2.66-9.36$ \\
\hline & Treatment-specific & Fixed & $+5.68^{\mathrm{a}}$ & $3.06-8.29$ & - & - \\
\hline & & Random & $+6.03^{\mathrm{a}}$ & $0.51-11.94$ & & \\
\hline \multirow[t]{6}{*}{ IVT-AFL 2q8 vs ranibizumab 0.5 mg T\&E } & None & Fixed & - & - & $+5.06^{\mathrm{a}}$ & $1.88-8.23$ \\
\hline & & Random & & & +5.07 & $-0.37-10.54$ \\
\hline & Common (baseline BCVA) & Fixed & - & - & $+4.90^{\mathrm{a}}$ & $2.06-7.72$ \\
\hline & & Random & & & +5.15 & $-0.26-10.61$ \\
\hline & Treatment-specific & Fixed & - & - & - & - \\
\hline & & Random & & & & \\
\hline \multirow[t]{6}{*}{ IVT-AFL 2 q8 vs ranibizumab $0.5 \mathrm{mg}$ T\&E + laser } & None & Fixed & - & - & $+5.07^{\mathrm{a}}$ & $2.00-8.13$ \\
\hline & & Random & & & +3.80 & $-1.57-9.25$ \\
\hline & Common (baseline BCVA) & Fixed & - & - & $+3.65^{a}$ & $0.94-6.35$ \\
\hline & & Random & & & +3.90 & $-1.42-9.25$ \\
\hline & Treatment-specific & Fixed & - & - & - & - \\
\hline & & Random & & & & \\
\hline
\end{tabular}


Table 3 IPD-only and IPD and aggregate network results for proportion of patients gaining $\geq 10$ and $\geq 15$ ETDRS letters

\begin{tabular}{|c|c|c|c|c|c|c|}
\hline \multirow{2}{*}{ IPD-only network Comparison } & \multirow[b]{2}{*}{ Covariable adjustment } & \multirow[b]{2}{*}{ Model } & \multicolumn{2}{|c|}{ Gain $\geq 10$ ETDRS letters } & \multicolumn{2}{|c|}{ Gain $\geq 15$ ETDRS letters } \\
\hline & & & OR & $95 \% \mathrm{Crl}$ & OR & $95 \% \mathrm{Crl}$ \\
\hline \multirow[t]{6}{*}{ IVT-AFL 2q8 vs laser } & \multirow[t]{2}{*}{ None } & Fixed & $4.82^{\mathrm{a}}$ & $3.52-6.46$ & $4.97^{\mathrm{a}}$ & $3.32-7.26$ \\
\hline & & Random & $18.72^{\mathrm{a}}$ & $1.16-20.20$ & $5.76^{\mathrm{a}}$ & $1.97-12.24$ \\
\hline & \multirow[t]{2}{*}{ Common (baseline BCVA) } & Fixed & $4.96^{\mathrm{a}}$ & $3.57-6.73$ & $5.49^{\mathrm{a}}$ & $3.47-8.38$ \\
\hline & & Random & $60.90^{\mathrm{a}}$ & $1.06-26.91$ & $7.58^{\mathrm{a}}$ & $1.79-15.34$ \\
\hline & \multirow[t]{2}{*}{ Treatment-specific } & Fixed & $5.10^{\mathrm{a}}$ & $3.67-6.94$ & $5.64^{\mathrm{a}}$ & $3.55-8.66$ \\
\hline & & Random & $7.28^{\mathrm{a}}$ & $1.16-24.20$ & $534.90^{\mathrm{a}}$ & $1.85-19.79$ \\
\hline \multirow[t]{6}{*}{ IVT-AFL 2 q8 vs ranibizumab 0.5 mg PRN } & \multirow[t]{2}{*}{ None } & Fixed & $2.10^{\mathrm{a}}$ & $1.19-3.45$ & $2.59^{\mathrm{a}}$ & $1.29-4.69$ \\
\hline & & Random & $93,680.00$ & $0.10-29.60$ & 10.68 & $0.53-14.63$ \\
\hline & \multirow[t]{2}{*}{ Common (baseline BCVA) } & Fixed & $2.09^{a}$ & $1.15-3.48$ & $2.85^{\mathrm{a}}$ & $1.35-5.35$ \\
\hline & & Random & 32.25 & $0.08-42.16$ & 59.97 & $0.37-20.4$ \\
\hline & \multirow[t]{2}{*}{ Treatment-specific } & Fixed & $1.99^{\mathrm{a}}$ & $1.07-3.38$ & $3.06^{\mathrm{a}}$ & $1.43-5.85$ \\
\hline & & Random & 594.80 & $0.14-43.29$ & 2056.00 & $0.43-39.48$ \\
\hline \multirow[t]{7}{*}{ IVT-AFL 2 q8 vs ranibizumab 0.5 mg PRN + laser } & \multirow[t]{2}{*}{ None } & Fixed & $3.04^{\mathrm{a}}$ & $1.82-4.77$ & $2.74^{a}$ & $1.44-4.78$ \\
\hline & & Random & 22.24 & $0.29-31.29$ & 5.93 & $0.76-13.29$ \\
\hline & \multirow[t]{2}{*}{ Common (baseline BCVA) } & Fixed & $2.92^{\mathrm{a}}$ & $1.70-4.68$ & $2.88^{\mathrm{a}}$ & $1.44-5.23$ \\
\hline & & Random & 969.00 & $0.28-41.36$ & 10.13 & $0.59-16.11$ \\
\hline & \multirow[t]{3}{*}{ Treatment-specific } & Fixed & $2.99^{\mathrm{a}}$ & $1.73-4.81$ & $2.99^{\mathrm{a}}$ & $1.48-5.44$ \\
\hline & & Random & 22.32 & $0.37-47.51$ & 52.20 & $0.60-23.38$ \\
\hline & & & \multicolumn{2}{|c|}{ Gain $\geq 10$ ETDRS letters } & \multicolumn{2}{|c|}{ Gain $\geq 15$ ETDRS letters } \\
\hline IPD and aggregate network Comparison & Covariable adjustment & Model & OR & $95 \% \mathrm{Crl}$ & OR & $95 \% \mathrm{Crl}$ \\
\hline \multirow[t]{4}{*}{ IVT-AFL 2q8 vs laser } & \multirow[t]{2}{*}{ None } & Fixed & $4.82^{\mathrm{a}}$ & $3.52-6.45$ & $4.97^{\mathrm{a}}$ & $3.32-7.28$ \\
\hline & & Random & $5.06^{\mathrm{a}}$ & $2.39-9.49$ & $5.03^{\mathrm{a}}$ & $3.09-7.88$ \\
\hline & \multirow[t]{2}{*}{ Common (baseline BCVA) } & Fixed & $4.93^{\mathrm{a}}$ & $3.55-6.72$ & $5.58^{\mathrm{a}}$ & $3.52-8.58$ \\
\hline & & Random & $5.27^{\mathrm{a}}$ & $2.44-9.91$ & $5.62^{\mathrm{a}}$ & $3.28-9.15$ \\
\hline \multirow[t]{4}{*}{ IVT-AFL 2q8 vs ranibizumab 0.5 mg PRN } & \multirow[t]{2}{*}{ None } & Fixed & $1.72^{\mathrm{a}}$ & $1.11-2.53$ & $2.07^{\mathrm{a}}$ & $1.19-3.38$ \\
\hline & & Random & 1.76 & $0.62-3.94$ & $2.08^{\mathrm{a}}$ & $1.06-3.70$ \\
\hline & \multirow[t]{2}{*}{ Common (baseline BCVA) } & Fixed & $1.71^{\mathrm{a}}$ & $1.09-2.56$ & $2.28^{\mathrm{a}}$ & $1.25-3.85$ \\
\hline & & Random & 1.79 & $0.63-4.06$ & $2.30^{\mathrm{a}}$ & $1.12-4.20$ \\
\hline IVT-AFL 2 q8 vs ranibizumab $0.5 \mathrm{mg}$ PRN + laser & None & Fixed & $2.01^{\mathrm{a}}$ & $1.32-2.94$ & $2.11^{\mathrm{a}}$ & $1.22-3.42$ \\
\hline & & Random & 2.18 & $0.81-4.78$ & $2.14^{\mathrm{a}}$ & $1.12-3.76$ \\
\hline & Common (baseline BCVA) & Fixed & $1.96^{\mathrm{a}}$ & $1.26-2.92$ & $2.27^{\mathrm{a}}$ & $1.26-3.83$ \\
\hline & & Random & 2.20 & $0.81-4.90$ & $2.31^{\mathrm{a}}$ & $1.15-4.20$ \\
\hline IVT-AFL 2q8 vs ranibizumab 0.5 mg T\&E & None & Fixed & 1.57 & $0.76-2.86$ & 1.78 & $0.76-3.55$ \\
\hline & & Random & 1.88 & $0.30-6.26$ & 1.86 & $0.63-4.18$ \\
\hline & Common (baseline BCVA) & Fixed & 1.55 & $0.75-2.85$ & 1.96 & $0.81-4.00$ \\
\hline & & Random & 1.95 & $0.32-6.47$ & 2.04 & $0.67-4.77$ \\
\hline IVT-AFL 2 q8 vs ranibizumab $0.5 \mathrm{mg}$ T\&E + laser & None & Fixed & $2.41^{\mathrm{a}}$ & $1.15-4.48$ & $3.22^{\mathrm{a}}$ & $1.32-6.68$ \\
\hline & & Random & 2.88 & $0.47-9.68$ & $3.35^{\mathrm{a}}$ & $1.09-7.84$ \\
\hline & Common (baseline BCVA) & Fixed & $2.39^{\mathrm{a}}$ & $1.13-4.48$ & $3.56^{\mathrm{a}}$ & $1.42-7.53$ \\
\hline & & Random & 3.02 & $0.48-9.87$ & $3.73^{\mathrm{a}}$ & $1.16-8.88$ \\
\hline
\end{tabular}


Table 4 IPD-only and IPD and aggregate network results for patients losing $\geq 10$ and $\geq 15$ ETDRS letters

\begin{tabular}{|c|c|c|c|c|c|c|}
\hline \multirow{2}{*}{ IPD-only network Comparison } & \multirow[b]{2}{*}{ Covariable adjustment } & \multirow[b]{2}{*}{ Model } & \multicolumn{2}{|c|}{ Loss $\geq 10$ ETDRS letters } & \multicolumn{2}{|c|}{ Loss $\geq 15$ ETDRS letters } \\
\hline & & & OR & $95 \% \mathrm{Crl}$ & OR & $95 \% \mathrm{Crl}$ \\
\hline \multirow[t]{6}{*}{ IVT-AFL vs laser } & \multirow[t]{2}{*}{ None } & Fixed & $0.06^{\mathrm{a}}$ & $0.02-0.12$ & $0.04^{\mathrm{a}}$ & $0.01-0.112$ \\
\hline & & Random & $0.11^{\mathrm{a}}$ & $0.01-0.29$ & $0.22^{\mathrm{a}}$ & $0.00-0.28$ \\
\hline & \multirow[t]{2}{*}{ Common (baseline BCVA) } & Fixed & $0.06^{\mathrm{a}}$ & $0.02-0.12$ & $0.04^{\mathrm{a}}$ & $0.00-0.11$ \\
\hline & & Random & $0.19^{\mathrm{a}}$ & $0.01-0.27$ & $0.07^{\mathrm{a}}$ & $0.00-0.24$ \\
\hline & \multirow[t]{2}{*}{ Treatment-specific } & Fixed & $0.05^{\mathrm{a}}$ & $0.02-0.11$ & $0.02^{\mathrm{a}}$ & $0.00-0.08$ \\
\hline & & Random & $0.08^{\mathrm{a}}$ & $0.01-0.24$ & $0.04^{\mathrm{a}}$ & $0.00-0.16$ \\
\hline \multirow[t]{6}{*}{ IVT-AFL vs ranibizumab $0.5 \mathrm{mg}$ PRN } & \multirow[t]{2}{*}{ None } & Fixed & 0.37 & $0.07-1.22$ & 0.51 & $0.02-2.51$ \\
\hline & & Random & 92.49 & $0.01-8.70$ & $278,400.00$ & $0.00-39.31$ \\
\hline & \multirow[t]{2}{*}{ Common (baseline BCVA) } & Fixed & 0.37 & $0.07-1.24$ & 0.47 & $0.02-2.34$ \\
\hline & & Random & 483.00 & $0.01-6.20$ & 825.00 & $0.00-22.22$ \\
\hline & \multirow[t]{2}{*}{ Treatment-specific } & Fixed & 0.51 & $0.07-2.07$ & 0.45 & $0.00-2.59$ \\
\hline & & Random & 9.28 & $0.01-7.53$ & 300.00 & $0.00-15.68$ \\
\hline \multirow[t]{7}{*}{ IVT-AFL vs ranibizumab $0.5 \mathrm{mg}$ PRN + laser } & \multirow[t]{2}{*}{ None } & Fixed & $0.17^{a}$ & $0.04-0.44$ & $0.23^{a}$ & $0.02-0.91$ \\
\hline & & Random & 6.74 & $0.01-2.11$ & 74.93 & $0.00-7.32$ \\
\hline & \multirow[t]{2}{*}{ Common (baseline BCVA) } & Fixed & $0.18^{\mathrm{a}}$ & $0.04-0.47$ & $0.20^{\mathrm{a}}$ & $0.02-0.81$ \\
\hline & & Random & 12.89 & $0.01-1.75$ & 11.07 & $0.00-5.17$ \\
\hline & \multirow[t]{3}{*}{ Treatment-specific } & Fixed & $0.18^{\mathrm{a}}$ & $0.04-0.51$ & $0.13^{\mathrm{a}}$ & $0.00-0.65$ \\
\hline & & Random & 3.38 & $0.01-1.89$ & 5.42 & $0.00-3.04$ \\
\hline & & & \multicolumn{2}{|c|}{ Loss $\geq 10$ ETDRS letters } & \multicolumn{2}{|c|}{ Loss $\geq 15$ ETDRS letters } \\
\hline $\begin{array}{l}\text { IPD and aggregate network } \\
\text { Comparison }\end{array}$ & Covariable adjustment & Model & OR & $95 \% \mathrm{Crl}$ & OR & $95 \% \mathrm{Crl}$ \\
\hline \multirow[t]{4}{*}{ IVT-AFL 2q8 vs laser } & \multirow[t]{2}{*}{ None } & Fixed & $0.06^{\mathrm{a}}$ & $0.02-0.12$ & $0.04^{\mathrm{a}}$ & $0.00-0.12$ \\
\hline & & Random & $0.06^{\mathrm{a}}$ & $0.02-0.14$ & $0.04^{\mathrm{a}}$ & $0.00-0.14$ \\
\hline & \multirow[t]{2}{*}{ Common (baseline BCVA) } & Fixed & $0.06^{\mathrm{a}}$ & $0.02-0.12$ & $0.04^{\mathrm{a}}$ & $0.00-0.11$ \\
\hline & & Random & $0.06^{\mathrm{a}}$ & $0.02-0.14$ & $0.04^{\mathrm{a}}$ & $0.00-0.13$ \\
\hline \multirow[t]{4}{*}{ IVT-AFL 2q8 vs ranibizumab 0.5 mg PRN } & \multirow[t]{2}{*}{ None } & Fixed & $0.24^{\mathrm{a}}$ & $0.07-0.60$ & 0.31 & $0.02-1.15$ \\
\hline & & Random & $0.25^{\mathrm{a}}$ & $0.05-0.73$ & 0.36 & $0.02-1.49$ \\
\hline & \multirow[t]{2}{*}{ Common (baseline BCVA) } & Fixed & $0.24^{\mathrm{a}}$ & $0.07-0.59$ & 0.28 & $0.02-1.05$ \\
\hline & & Random & $0.25^{\mathrm{a}}$ & $0.05-0.74$ & 0.33 & $0.02-1.39$ \\
\hline IVT-AFL 2 q8 vs ranibizumab 0.5 mg PRN + laser & None & Fixed & $0.14^{\mathrm{a}}$ & $0.04-0.33$ & $0.11^{\mathrm{a}}$ & $0.01-0.37$ \\
\hline & & Random & $0.15^{\mathrm{a}}$ & $0.03-0.41$ & $0.13^{\mathrm{a}}$ & $0.01-0.47$ \\
\hline & Common (baseline BCVA) & Fixed & $0.14^{\mathrm{a}}$ & $0.04-0.33$ & $0.10^{\mathrm{a}}$ & $0.01-0.34$ \\
\hline & & Random & $0.15^{\mathrm{a}}$ & $0.03-0.41$ & $0.11^{\mathrm{a}}$ & $0.01-0.43$ \\
\hline IVT-AFL 2 q8 vs ranibizumab 0.5 mg T\&E & None & Fixed & $0.15^{\mathrm{a}}$ & $0.01-0.75$ & 0.33 & $0.00-1.94$ \\
\hline & & Random & $0.19^{\mathrm{a}}$ & $0.00-0.96$ & 0.81 & $0.00-3.00$ \\
\hline & Common (baseline BCVA) & Fixed & $0.15^{\mathrm{a}}$ & $0.00-0.73$ & 0.30 & $0.00-1.74$ \\
\hline & & Random & $0.21^{\mathrm{a}}$ & $0.00-0.97$ & 0.54 & $0.00-2.67$ \\
\hline IVT-AFL 2 q8 vs ranibizumab 0.5 mg T\&E + laser & None & Fixed & $222,500.00$ & $0.03-607.2$ & $201,200.00$ & $0.02-671.3$ \\
\hline & & Random & $325,000.00$ & $0.02-589.9$ & $130,000.00$ & $0.01-751.0$ \\
\hline & Common (baseline BCVA) & Fixed & $470,400.00$ & $0.03-555.1$ & $3,311,000.00$ & $0.02-592.0$ \\
\hline & & Random & $662,700.00$ & $0.02-622.8$ & $2,877,000.00$ & $0.01-775.5$ \\
\hline
\end{tabular}

aIVT-AFL showed statistical superiority to the comparator as 95\% Crl ranges did not cross 1 


\section{Discussion}

It is important for physicians and policymakers to compare the relative efficacy of DME treatments using the most robust methods available. Although direct headto-head comparative trials provide stronger internal validity than indirect comparisons, there are a small number of these trials in DME (eg, DRCR.net Protocol T) $[4,8]$, and there are no direct randomised comparative trial outcomes of IVT-AFL 2q8 and ranibizumab $0.5 \mathrm{mg}$ (any regimen) available. The objective of this NMA was to perform an updated indirect comparison of IVT-AFL 2q8 versus relevant comparators, including ranibizumab 0.5 mg, from a previous publication [9]. Notably, this NMA incorporated IPD where available and adjusted for baseline BCVA, which is a known and important treatment effect modifier. To our knowledge, this is also the first NMA in DME that adjusted for any imbalance of treatment effect modifiers using IPD. Previous analyses that used only aggregate data are prone to ecological bias.

In the IPD and aggregate random-effects models, IVT-AFL 2q8 (after 5 monthly loading doses) showed strong evidence of superiority versus laser and ranibizumab $0.5 \mathrm{mg}$ PRN with/without laser for mean change in BCVA from baseline and for gain of $\geq 15$ ETDRS letters. IVT-AFL 2q8 also showed strong evidence of superiority for reducing $\geq 10$ ETDRS letter losses versus ranibizumab $0.5 \mathrm{mg}$ PRN with/without laser and versus ranibizumab $0.5 \mathrm{mg}$ T\&E. These results were consistent regardless of whether adjustment was made for baseline BCVA.

The BCVA results are similar to those in the previously published NMA that included fewer trials and did not include IPD [9]. In this previous NMA, there was an increase in mean BCVA at 12 months with IVT-AFL 2q8 over ranibizumab $0.5 \mathrm{mg}$ PRN of 4.67 letters $(95 \% \mathrm{CrI}$ $1.85-7.52)$ in the random-effects Bayesian model (10 trials). However, there was no significant difference between IVT-AFL 2q8 versus ranibizumab $0.5 \mathrm{mg}$ PRN for gain of $\geq 15$ ETDRS letters (OR 1.87, 95\% Crl 0.87-4.16) or loss of $\geq 10$ ETDRS letters (OR 0.26, 95\% Crl 0.05-1.31) in the random-effects Bayesian model (6 trials). The BCVA results are also consistent with those in a recent NMA that used an aggregate data approach [34]. This NMA of 21 trials (4307 eyes), which compared 11 different DME interventions including IVT-AFL and ranibizumab $0.5 \mathrm{mg}$, showed that IVT-AFL was the most favourable treatment at 12 months with respect to improvements in BCVA (OR 8.19, 95\% CrI 5.07-11.96) and central macular thickness (OR -110.83, 95\% CrI -190.25 to - 35.27) [34].

The availability and use of IPD can be important for ensuring that NMAs provide more robust adjustment for differences in between-trial baseline BCVA. In our NMA, we showed that adjustment for baseline BCVA did not affect the results in any model, but a lower
BCVA at baseline was associated with a greater response to treatment. These findings are comparable with those observed in DRCR.net Protocol $\mathrm{T}$ [4]. In DRCR.net Protocol $\mathrm{T}$, the mean change in visual acuity score (VAS) at 12 months was greater in patients with a lower BCVA score at baseline; for patients with baseline VAS of 74-78, the mean change in VAS was $\sim 7$ letters (IVT-AFL $2 \mathrm{mg}$ ) and $\sim 6.5$ letters (ranibizumab $0.3 \mathrm{mg}$ ) at 12 months, and for patients with baseline VAS of 24-53 letters the mean change in VAS was $\sim 25$ letters (IVT-AFL $2 \mathrm{mg}$ ) and $\sim 17.5$ letters (ranibizumab $0.3 \mathrm{mg}$ ) [4]. Similar findings were observed in a post hoc analysis of the VISTA-DME and VIVID-DME trials, IVT-AFL 2q8 treatment resulted in slightly better 12 -month visual acuity outcomes in patients with baseline BCVA of $\geq 39$ to $\leq 60$ letters compared with those with a baseline BCVA of $\geq 61$ to $\leq 73$ letters; the mean change with IVT-AFL $2 \mathrm{q} 8$ at 12 months was 11.3 ETDRS letters (VIVID-DME) and 11.4 ETDRS letters (VISTA-DME) (baseline BCVA of $\geq 39$ to $\leq 60$ letters) and 8.6 ETDRS letters (VIVID-DME) and 9.5 ETDRS letters (VISTA-DME) (baseline BCVA of $\geq 61$ to $\leq 73$ letters) [35].

The comparison of IVT-AFL 2q8 versus ranibizumab $0.5 \mathrm{mg}$ (PRN and T\&E) also provides a useful complement to the direct comparative evidence of IVT-AFL 2 mg versus ranibizumab $0.3 \mathrm{mg}$ from DRCR.net Protocol $\mathrm{T}[4,8]$. The EURETINA guidelines question the extent to which the slower effect of ranibizumab seen in DRCR.net Protocol T compared with IVT-AFL is attributable to the lower dose used [36]. This outcome is consistent with those in other published NMAs [9, 37]. In an updated Cochrane analysis of 24 trials (6007 patients with DME) [37], DME patients receiving ranibizumab ( $0.3 \mathrm{mg}$ and $0.5 \mathrm{mg}$ monthly) were less likely to gain $\geq 3$ lines of visual acuity at 1 year compared with IVT-AFL (risk ratio [RR] $0.75,95 \%$ CI 0.60-0.94). At 1 year, the visual acuity and CRT were worse with ranibizumab compared with IVT-AFL (mean difference in visual acuity: $0.08 \log$ MAR, 95\% CI 0.05-0.11; mean difference in CRT: $39 \mu \mathrm{m}, 95 \%$ CI 2-76). It should be noted that ranibizumab $0.3 \mathrm{mg}$ and $0.5 \mathrm{mg}$ monthly were merged into 1 group as no heterogeneity was suspected between studies using these regimens in this Cochrane analysis. These authors concluded that the difference between IVT-AFL and ranibizumab was consistent with indirect evidence using ranibizumab $0.5 \mathrm{mg}$.

Despite the robust approach used in the current analysis, there are still some limitations associated with this updated NMA. We did not consider safety outcomes or longer-term efficacy outcomes, and there was limited availability of IPD in the public domain. Outcomes could also have been improved with the replacement of aggregate data from some trials (such as DRCR.net Protocol T) with IPD; however, the availability of IPD from 
DRCR.net Protocols I and $\mathrm{J}$ in addition to VIVID-DME, VISTA-DME, and VIVID-EAST enabled sufficient IPD to be included.

\section{Conclusions}

This NMA, which incorporated aggregate data and IPD to improve model robustness, consistently showed evidence of superiority of IVT-AFL $2 \mathrm{q} 8$ to laser and ranibizumab $0.5 \mathrm{mg}$ PRN with/without laser for mean change in BCVA, gain of $\geq 15$ ETDRS letters, and loss of $\geq 10$ ETDRS letters at 12 months. IVT-AFL $2 \mathrm{q} 8$ was also superior to ranibizumab $0.5 \mathrm{mg} \mathrm{T} \& \mathrm{E}$ for loss of $\geq 10$ ETDRS letters. These efficacy results were consistent irrespective of adjustment for baseline BCVA. Comparison of IVT-AFL 2q8 versus ranibizumab $0.5 \mathrm{mg}$ PRN and T\&E provides a useful complement to the direct comparative evidence of IVT-AFL $2 \mathrm{mg}$ versus ranibizumab $0.3 \mathrm{mg}$ given in DRCR.net Protocol T. It is hoped that these data will be of additional benefit to those involved in the care of DME patients and to policymakers interested in developing future NMAs.

\section{Additional files}

Additional file 1: Appendix 1. Statistical methods and covariable adjustments. (DOCX 57 kb)

Additional file 2: Appendix 2. Treatment regimens classified and included in the NMA. (DOCX $64 \mathrm{~kb}$ )

Additional file 3: Appendix 3. Overview of outcomes of studies included in the NMA. (DOCX $68 \mathrm{~kb})$

\section{Abbreviations}

2q8: 2 mg bimonthly; BCVA: Best-corrected visual acuity; Cl: Confidence interval; Crl: Credible interval; CRT: Central retinal thickness; DME: Diabetic macular edema; DRCR.net: Diabetic Retinopathy Clinical Research Network; ETDRS: Early Treatment Diabetic Retinopathy Study; IPD: Individual patientlevel data; IVT-AFL: Intravitreal aflibercept; NMA: Network meta-analysis; OR: Odds ratio; PICOS: Populations, interventions, comparators, outcomes and study design; PRN: As-needed; RCT: Randomised controlled trial; SD: Standard deviation; SE: Standard error; T\&E: Treat-and-extend; VAS: Visual acuity score; VEGF: Vascular endothelial growth factor

\section{Acknowledgements}

Medical writing assistance was provided by Corey Eagan, MPH, and Sam Phillips, PhD, of PAREXEL, and was funded by Bayer Pharma AG.

\section{Funding}

The analysis was funded by Bayer Pharma AG, who were involved in the study design, data collection, data analysis, and manuscript writing.

\section{Availability of data and materials}

The datasets used and/or analysed during the current study are available from the corresponding author on reasonable request.

\section{Authors' contributions}

$\mathrm{NH}, \mathrm{TR}, \mathrm{IC}, \mathrm{FR}$, and DM were involved in the design, analysis, interpretation of data, and manuscript preparation. JFK and PK were involved in the interpretation of data and manuscript preparation. All authors were involved with manuscript development and have read and approved the final version.

\section{Ethics approval and consent to participate}

All included trials had protocols approved by relevant country- and trialspecific institutional review boards/independent ethics committees.

\section{Consent for publication}

Not applicable.

\section{Competing interests}

The authors have the following financial competing interests to declare: Dominic Muston is a former employee of Bayer.

Jean-Francois Korobelnik is a consultant for Alcon, Allergan, Bayer, Kanghong, Novartis, and Roche.

Tim Reason, Ismini Chatzitheofilou, and Fay Ryan are employees of QuintilesIMS, which was funded by Bayer to undertake the project on which this article is based.

Neil Hawkins is a consultant for Bayer.

Peter Kaiser is a consultant for Alcon, Allergan, Bayer, Kanghong, Novartis, and Regeneron.

\section{Publisher's Note}

Springer Nature remains neutral with regard to jurisdictional claims in published maps and institutional affiliations.

\section{Author details}

${ }^{1}$ Bayer US LLC, Whippany, NJ, USA. ²Service d'ophtalmologie CHU, Bordeaux, France. ${ }^{3}$ University of Bordeaux, Inserm, Bordeaux Population Health Research Center, team LEHA, Bordeaux, France. ${ }^{4}$ QuintilesIMS, London, UK. ${ }^{5}$ University of Glasgow, Glasgow, UK. ${ }^{6} \mathrm{Cole}$ Eye Institute, Cleveland, $\mathrm{OH}, \mathrm{USA}$.

Received: 22 June 2018 Accepted: 10 December 2018

Published online: 27 December 2018

\section{References}

1. Au A, Singh RP. A multimodal approach to diabetic macular edema. J Diabetes Complicat. 2016;30:545-53.

2. Fogli S, Mogavero S, Egan CG, Del Re M, Danesi R. Pathophysiology and pharmacological targets of VEGF in diabetic macular edema. Pharmacol Res. 2016;103:149-57.

3. Varma R, Bressler NM, Doan QV, et al. Visual impairment and blindness avoided with ranibizumab in Hispanic and non-Hispanic whites with diabetic macular edema in the United States. Ophthalmology. 2015;122: 982-9.

4. Diabetic Retinopathy Clinical Research Network, Wells JA, Glassman AR, et al. Aflibercept, bevacizumab, or ranibizumab for diabetic macular edema. N Engl J Med. 2015;372:1193-203.

5. Mitchell P, Bandello F, Schmidt-Erfurth U, et al. The RESTORE study: ranibizumab monotherapy or combined with laser versus laser monotherapy for diabetic macular edema. Ophthalmology. 2011;118:615-25.

6. Nguyen QD, Brown DM, Marcus DM, et al. Ranibizumab for diabetic macular edema: results from 2 phase III randomized trials: RISE and RIDE. Ophthalmology. 2012;119:789-801.

7. Korobelnik JF, Do DV, Schmidt-Erfurth U, et al. Intravitreal aflibercept for diabetic macular edema. Ophthalmology. 2014;121:2247-54.

8. Wells JA, Glassman AR, Ayala AR, et al. Aflibercept, bevacizumab, or ranibizumab for diabetic macular edema: two-year results from a comparative effectiveness randomized clinical trial. Ophthalmology. 2016; 123:1351-9.

9. Korobelnik JF, Kleijnen J, Lang SH, et al. Systematic review and mixed treatment comparison of intravitreal aflibercept with other therapies for diabetic macular edema (DME). BMC Ophthalmol. 2015;15:52.

10. Regnier S, Malcolm W, Allen F, Wright J, Bezlyak V. Efficacy of anti-VEGF and laser photocoagulation in the treatment of visual impairment due to diabetic macular edema: a systematic review and network meta-analysis. PLoS One. 2014;9:e102309.

11. Virgili G, Parravano M, Menchini F, Evans JR. Anti-vascular endothelial growth factor for diabetic macular oedema. Cochrane Database Syst Rev. 2014:CD007419.

12. Jansen JP, Naci H. Is network meta-analysis as valid as standard pairwise meta-analysis? It all depends on the distribution of effect modifiers. BMC Med. 2013;11:159. 
13. Dias S, Sutton AJ, Welton NJ, Ades AE. Evidence synthesis for decision making 3: heterogeneity--subgroups, meta-regression, bias, and biasadjustment. Med Decis Mak. 2013;33:618-40.

14. European Medicines Agency. European Medicines Agency policy on publication of clinical data for medicinal products for human use. . http:// www.ema.europa.eu/docs/en_GB/document_library/Other/2014/10/ WC500174796.pdf. Accessed 7 May 2018.

15. Higgins JP, Thompson SG, Deeks JJ, Altman DG. Measuring inconsistency in meta-analyses. BMJ. 2003;327:557-60.

16. Dias S, Welton NJ, Sutton AJ, Ades AE. A generalised linear modelling framework for pairwise and network meta-analysis of randomised controlled trials. NICE DSU technical support document no. 2. In: London; 2014.

17. Misiuk-Hojlo MB, D.M.; Katz, T. Impact of baseline diabetic retinopathy severity scale score on visual outcomes in VIVID-DMENISTA-DME. Presented at: the 7th world congress on controversies in ophthalmology (COPHy); 31 march - 02 April 2016; Warsaw, Poland.

18. Do DV, Nguyen QD, Vitti $R$, et al. Intravitreal aflibercept injection in diabetic macular edema patients with and without prior anti-vascular endothelial growth factor treatment: outcomes from the phase 3 program. Ophthalmology. 2016;123:850-7.

19. Gomez A, Sanchez JG, Brown D, Korobelnik J-F. Effect of baseline glycated haemoglobin on treatment outcomes with intravitreal aflibercept in VIVIDDME and VISTA-DME. Presented at: pan-American Association of Ophthalmology Congress; 4-8 august 2015; Bogotá, Colombia.

20. Evans M, Crane M, Katz T. Effect of baseline hemoglobin A1C and on-treatment blood pressure at week 100 in the VIVID-DME and VISTA-DME studies. Presented at: international diabetes federation 2015 - the world diabetes congress; 30 November - 04 December 2015; Vancouver, Can Underwrit.

21. Moshfeghi A. Outcomes before and after cataract surgery in patients treated for diabetic macular edema (DME) in VISTA and VIVID. Presented at: American Society of Retina Specialists 34th Annual Meeting; 10-14 August 2016; San Francisco, California, USA.

22. Metzig C, Gillies M, Ogura Y, Lu C, Katz TA. Effect of baseline central retinal thickness and best-corrected visual acuity on VA outcomes in VIVD-DME and VISTA-DME. Poster 106. Presented at: Royal College of ophthalmologists annual congress; 23-26 may 2016; Birmingham, UK.

23. Welton NJ, Sutton AJ, Cooper NJ, Abrams KR, Ades AE, eds. Evidence synthesis for decision making in healthcare. West Sussex, UK: John Wiley \& Sons, Ltd.; 2012

24. Hawkins N, Scott DA, Woods B. 'Arm-based' parameterization for network meta-analysis. Res Synth Methods. 2016;7:306-13.

25. Hoaglin DC, Hawkins N, Jansen JP, et al. Conducting indirect-treatmentcomparison and network-meta-analysis studies: report of the ISPOR task force on indirect treatment comparisons good research practices: part 2. Value Health. 2011;14:429-37.

26. Jansen JP, Fleurence $R$, Devine $B$, et al. Interpreting indirect treatment comparisons and network meta-analysis for health-care decision making: report of the ISPOR task force on indirect treatment comparisons good research practices: part 1. Value Health. 2011;14:417-28.

27. Diabetic Retinopathy Clinical Research Network, Elman MJ, Aiello LP, et al Randomized trial evaluating ranibizumab plus prompt or deferred laser or triamcinolone plus prompt laser for diabetic macular edema. Ophthalmology 2010;117:1064-1077 e35.

28. Diabetic Retinopathy Clinical Research Network, Googe J, Brucker AJ, et al. Randomized trial evaluating short-term effects of intravitreal ranibizumab or triamcinolone acetonide on macular edema after focal/grid laser for diabetic macular edema in eyes also receiving panretinal photocoagulation. Retina 2011;31:1009-27.

29. Comyn O, Sivaprasad S, Peto T, et al. A randomized trial to assess functional and structural effects of ranibizumab versus laser in diabetic macular edema (the LUCIDATE study). Am J Ophthalmol. 2014;157:960-70.

30. Prunte C, Fajnkuchen F, Mahmood S, et al. Ranibizumab 0.5 mg treat-andextend regimen for diabetic macular oedema: the RETAIN study. $\mathrm{Br} J$ Ophthalmol. 2016;100:787-95.

31. Ishibashi T, Li X, Koh A, et al. The REVEAL study: ranibizumab monotherapy or combined with laser versus laser monotherapy in Asian patients with diabetic macular edema. Ophthalmology. 2015;122:1402-15.

32. Clinicaltrials.gov. Safety, efficacy and cost-efficacy of ranibizumab (monotherapy or combination with laser) in the treatment of diabetic macular edema (DME) (RESPOND). NCT01135914. . https://www.clinicaltrials. gov/ct2/show/NCT01135914?term=RESPOND\&cond=DME\&rank=1. Accessed 7 May 2018
33. Clinicaltrials.gov. Efficacy and safety of VEGF Trap Eye in diabetic macular edema (DME) with central involvement (VIVID EAST). NCT01783886. . https:// www.clinicaltrials.gov/ct2/show/NCT01783886?term=VIVID+EAST\&rank=1. Accessed 7 May 2018

34. Zhang L, Wang W, Gao Y, Lan J, Xie L. The efficacy and safety of current treatments in diabetic macular edema: a systematic review and network meta-analysis. PLoS One. 2016;11:e0159553.

35. Lorenz K. Evaluation of intravitreal aflibercept for treatment of diabetic macular edema: visual acuity subgroups in VIVID-DME and VISTA-DME. Invest Ophthalmol Vis Sci. 2014;55:5054.

36. Schmidt-Erfurth U, Garcia-Arumi J, Bandello F, et al. Guidelines for the management of diabetic macular edema by the European Society of Retina Specialists (EURETINA). Ophthalmologica. 2017;237:185-222.

37. Virgili G, Parravano M, Evans JR, Gordon I, Lucenteforte E. Anti-vascular endothelial growth factor for diabetic macular oedema: a network metaanalysis. Cochrane Database Syst Rev. 2017;6:CD007419.
Ready to submit your research? Choose BMC and benefit from:

- fast, convenient online submission

- thorough peer review by experienced researchers in your field

- rapid publication on acceptance

- support for research data, including large and complex data types

- gold Open Access which fosters wider collaboration and increased citations

- maximum visibility for your research: over $100 \mathrm{M}$ website views per year

At BMC, research is always in progress.

Learn more biomedcentral.com/submissions 